\title{
ENERGY EFFICIENT MODEL FOR DEPLOYING Wireless Body AREA Networks USINg MulTi- HOP NETWORK TOPOLOGY
}

\author{
Robert C. Chepkwony ${ }^{1}$, John O. Gwendo ${ }^{2}$, Peter K. Kemei ${ }^{3}$ \\ ${ }^{1}$ Digital School of Virtual and Open Learning, Kenyatta University, Nairobi, Kenya \\ ${ }^{2}$ Technology Education Department, Moi University, Eldoret, Kenya, \\ ${ }^{3}$ Computer Science Department, Faculty of Science, Egerton University, Nakuru, Kenya
}

\begin{abstract}
Wireless body area networks (WBANs) offers a lot of application opportunities in the area of health care. Recent developments in sensors and radio communication technologies have motivated many researchers to design WBAN systems for application in healthcare provision. Power consumption is still a limiting factor in realizing a WBAN with a very long lifetime.
\end{abstract}

In order for wireless body area networks to ensure widespread use and adoption, some of the design constraints should be solved to promote uptake and meet social expectations. As a result, design of energy efficient WBANs is required to enhance battery life at the same time ensure that sensor nodes are small enough to be conveniently worn or implanted in the body. Energy consumption in WBANs happens during sensing, processing and communication.

This research focused on designing an energy efficient model during communication between sensors. The parameters were simulated and implemented using MATLAB and Simulink simulation software. The sensors are randomly localized on a plane and distance between them calculated.

The model uses a relay between the sensors and the coordinator to reduce power consumption by sensors during signal transmission. The relay is dedicated to retransmitting signals only.

\section{KEYWORDS}

Wireless Body Area Network (WBAN), Energy efficiency, Sensor, MATLAB, Relay.

\section{INTRODUCTION}

WBAN is a wireless network which enables communication among sensor nodes operating on the body surface or inside the human body to collect information on various body parameters and even motions. In the deployment of WBAN networks, communication between the sensor devices is of critical design consideration. Communication between sensor nodes needs to consume minimal power and offer high reliability. In the recent past, a lot of research in the area of WBANs has been directed to areas like sensor circuitry, minute sensor design, processing of 
International Journal of Wireless \& Mobile Networks (IJWMN) Vol. 7, No. 5, October 2015

signals by the sensors protocols in WBAN implantation and other related issues, (Min Chen. et al, 2011).

Star topology is associated with high power consumption in relaying data over long range singlehop point-to-point links. The issue of on-body sensor movement, wireless connection links which are unreliable and changing from time to time and the importance of reliability and fast data signal transmission at minimal energy use are some challenges facing deployment of WBANs (Wout J et al, 2011).

Recent developments in reduced power wireless transmission, communication and signal processing have triggered enormous attention in the deployment and design of wireless technology in healthcare and biomedical research, as well as WBANs. Among the issues of utmost importance in WBANs is low power consumed by the circuitry of the sensor, signal processing and transmission of the data. The sensor nodes implanted in or worn on the body have small battery capacity or can get little energy from their surroundings. Thus to prolong the life of the network, mechanisms to conserve energy has to be adopted. As such efforts towards design of sensors which uses less power is alive among many researchers. Protocol design and network set up can be optimized to reduce energy used.

The use of wireless transceiver which consumes low power to minimize the amount of energy required normally will lead to a small coverage area in signal transmission. This implies that a multi-hop topology is one of the best design choices for implementing WBANs, (Majid N. et.al 2010).

A lot of research has been conducted towards developing power efficient WBANs. The focus has been on protocol design, energy efficient MAC schemes, use of wake up radio concept where the sensor sleeps when it is not communicating and wake up only when it has data to send ( $\mathrm{Al}$ Ameen et al, 2012)

(Wout $\mathrm{J}$ et al, 2012) investigated energy consumption in star and multi-hop topologies and found that multi-hop topology is associated with lower power consumption but the sensors closer to the coordinator uses a lot of energy to transmit signal from sensor nodes further away.

This research proposes a multi-hop topology model which uses a relay between the coordinator and distant sensor nodes. The relay is dedicated to transmit signals from distant nodes only while sensors closer to coordinator transmit directly to the coordinator.

\section{RELATED WORK}

This research aims to investigate the energy efficiency in multi-hop wireless body area networks. Some research has been carried out in Wireless Body Area Network (WBAN) architecture and energy consumption both in star and multi-hop topologies.

Multihop technology in wireless networking entails deployment without wired connections between communicating nodes. It involves devices communicating with signals hoping from one node to another until it reaches the destination device. Multihop relaying has been proven to be effective in increasing the capacity, coverage, reducing the call blocking probability and decreasing the per node transmission power. Govindan K. et al (2010), 
International Journal of Wireless \& Mobile Networks (IJWMN) Vol. 7, No. 5, October 2015

WBAN connects independent nodes (e.g., sensors and actuators) that are situated in the clothes, on the body or implanted under the skin of a person. The network typically extends over the whole human body and the nodes are connected through a wireless communication channel. According to the implementation, these nodes are placed in a star or multi-hop topology, (Wout J. etal 2011).

Energy consumption is a very key parameter in deployment of WBANs, it is therefore imperative that energy demands of WBANs should be as small as possible. WBAN consumes energy during sensing, communication and data processing. A lot of energy is consumed during communication, (Meenakshi B and Navroop 2011). In the last decade, techniques for low power design and compilation have been attracting a great deal of attention. Numerous techniques for reducing power consumption using either architecture and integrated circuit design approaches or compilation and operating system schemes have been proposed.

WBAN consist of various components which work together to collect, process, store, and transmit data from the body to specific points where the data is required for monitoring, interpretation or emergency response. The basic components are sensor nodes, control unit/sink and a gateway which connects the sensors to the wider network.

\section{Methodology ApProach}

\subsection{Proposed Methodological Approach}

In this research, simulation is chosen as the primary method to carry out the work. Simulation provides the required convenience, time and cheaper alternative to realize the aims of this research.

Simulation is a widely used technique for representing real-world systems, normally dynamic. The model mimics a real system allowing use of simulation tools and emulators running on computers to study various systems. There are a number of simulation tools which can be used to simulate various scenarios in WBANs studies.

Simulation is used in this research to enable the study of effects of various factors on signal transmission in WBAN.

\subsection{Conceptual Design}

The conceptual model is based on a typical WBAN for various applications, with focus on the factors which affect energy consumption during communication between nodes, relays and the sink.

The model outlines the relations between the various components, their attributes and interfaces. 


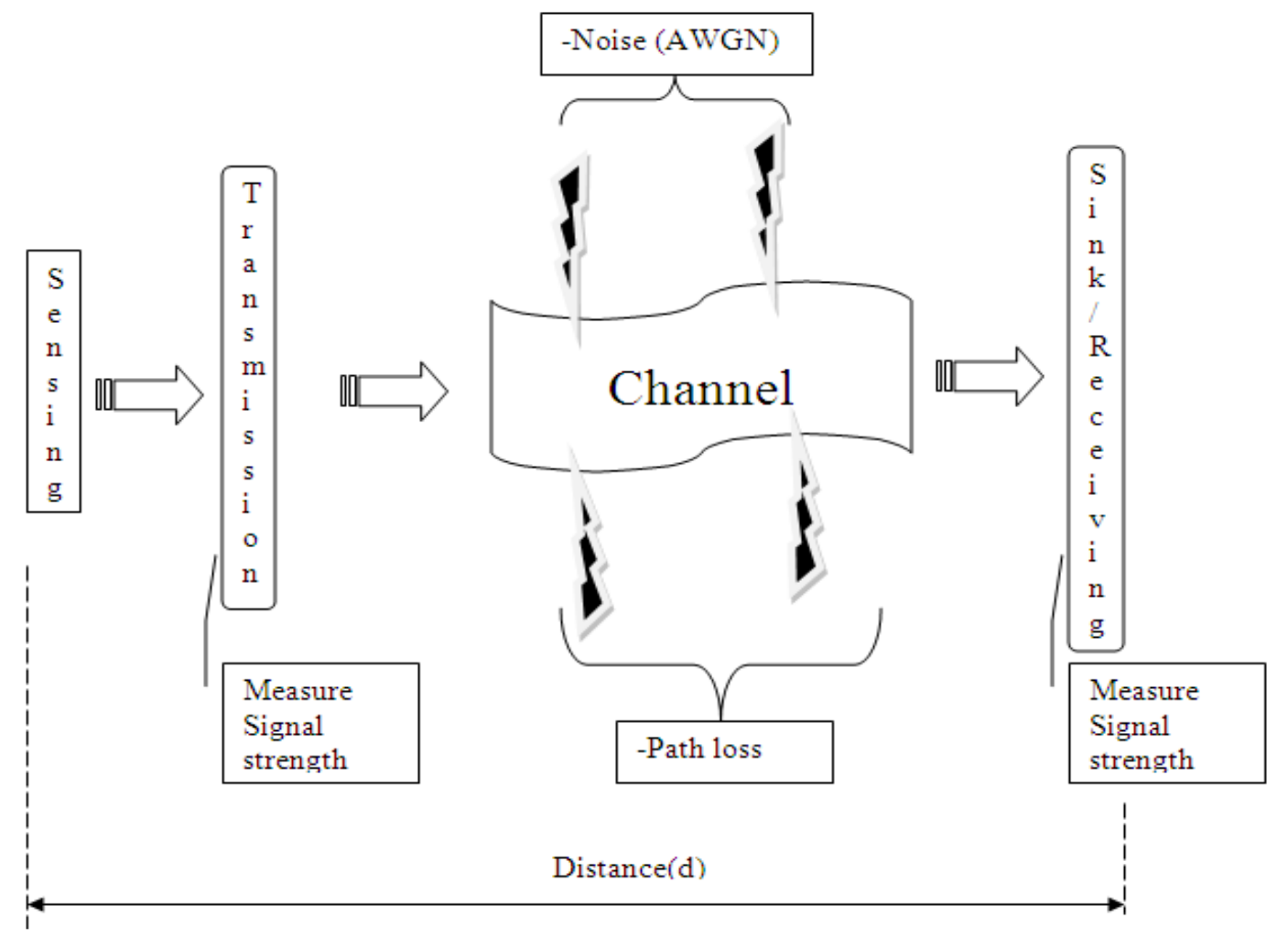

Figure 1: Conceptual Model

\subsubsection{Conceptual Model components}

\subsubsection{Sensing Unit}

This carries the sensors which collect data from various parts of the body. The sensors collect physical signals like pressure, movement, temperatures etcetera. The sensor picks these analog signals which are then transferred by the transmitter.

\subsubsection{Transmitter}

The transmission unit basically relays the collected data from the sensors to the receiver. The other parameters of importance in relaying of signals are distance and signal strength. The distance separating the transmitter and receiver is measured to get the optimal distance which will use minimum power to send the signal. The signal strength is measured at the transmitter and receiver to be able to establish the optimal distance.

\subsubsection{Channel}

The channel describes the parameters between the transmitter and the receiver. There are a number of channel parameters which will influence transmission of signals including: 
International Journal of Wireless \& Mobile Networks (IJWMN) Vol. 7, No. 5, October 2015

Noise: This is composed of any interference from the environment which may affect the signal transfer between the transmitter and the receiver. The kind of noise represented in this model is additive white Gaussian noise (AWGN).

Path loss: The path loss component is also factored in this model. This component is affected by distance and body movement. Path loss differs depending whether it is a line of sight or non line of sight communication.

\subsection{Data/Input Data}

\subsubsection{Node Location}

Node placement is achieved by using a MATLAB code to randomly generate node positions on $150 \mathrm{~cm}$ plane/graph. The choice of $150 \mathrm{~cm}$ graph was informed by using an average height of a person $150 \mathrm{~cm}$.

The nodes are randomly placed on the graph and the distance between the reference point (coordinator) and the nodes are calculated using the algorithm below. The $\mathrm{x}$ and $\mathrm{y}$ coordinates of the sensors are used to calculate the distance by applying Pythagorean Theorem.

If the distance between the coordinator and the sensor node is less than or equal to a predetermined distance $(\mathrm{R})$, the sensor transmits to the coordinator. Otherwise it transmits to the relay.

For all node pairs

Distance between nodes, $n_{i}, n_{j}$ and reference point (coordinator)

$X=\operatorname{abs}\left(x_{i}-x_{j}\right)$

$Y=a b s(y i-y j)$

Distance $=\operatorname{sqrt}\left(X^{2}+Y^{2}\right)$

If Distance Di,j $_{i}<=R$,

Connect $=$ coordinator

Else

Connect $=$ Relay 
International Journal of Wireless \& Mobile Networks (IJWMN) Vol. 7, No. 5, October 2015

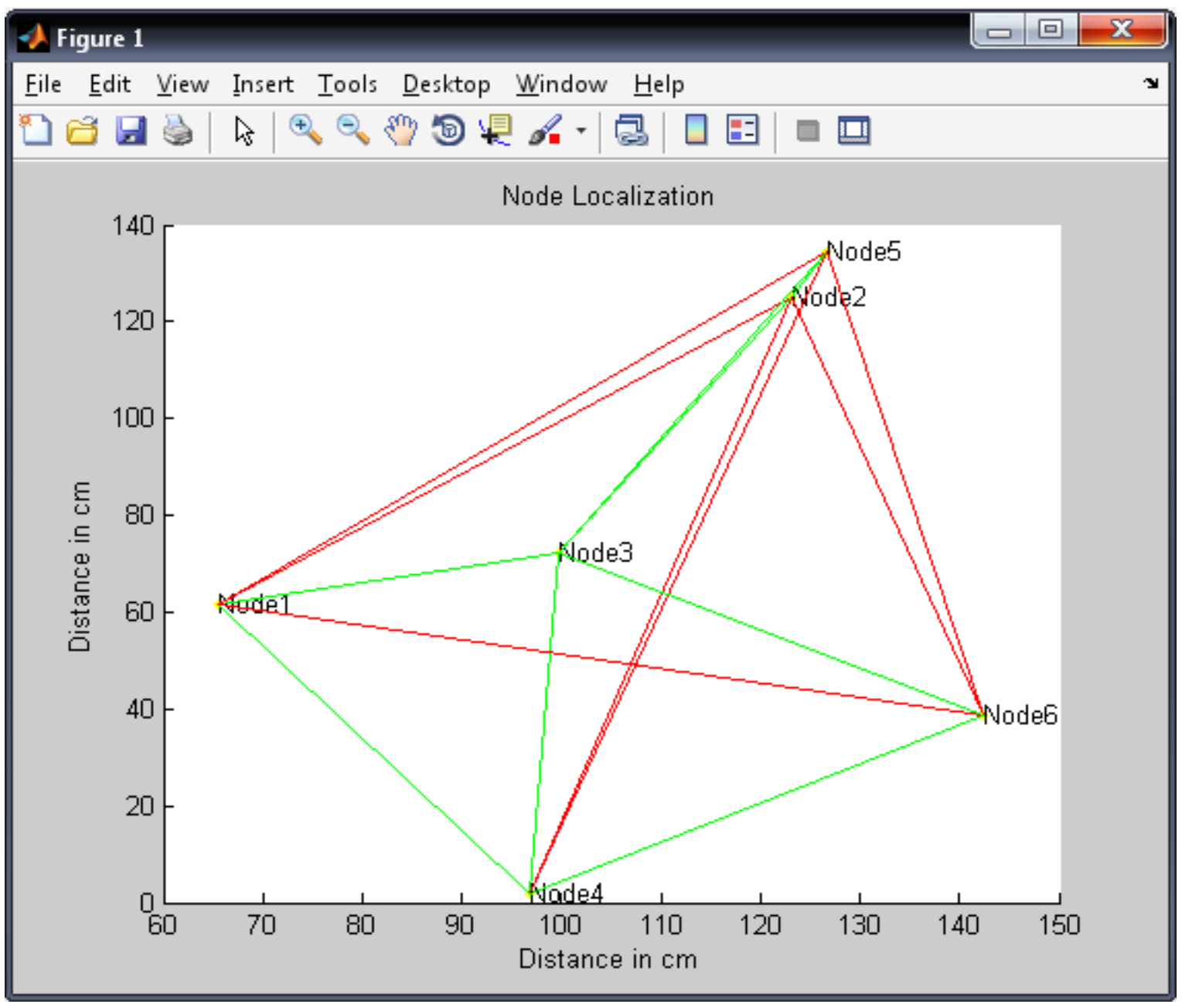

The distance between the nodes pairs from MATLAB simulation are shown in the table below:

Figure 2: Random Nodes Localization Chart 
International Journal of Wireless \& Mobile Networks (IJWMN) Vol. 7, No. 5, October 2015

Table 1: Distances Between Node Pairs

\begin{tabular}{|ll|}
\hline Node Pairs & Distance $(\mathrm{cm})$ \\
\hline $1-2$ & 85.6401 \\
\hline $1-3$ & 35.9114 \\
\hline $1-4$ & 67.6729 \\
\hline $1-5$ & 95.1741 \\
\hline $1-6$ & 80.4471 \\
\hline $2-3$ & 57.6061 \\
\hline $2-4$ & 125.9178 \\
\hline $2-5$ & 10.2681 \\
\hline $2-6$ & 88.5481 \\
\hline $3-4$ & 70.5833 \\
\hline $3-5$ & 67.8456 \\
\hline $3-6$ & 54.4830 \\
\hline $4-5$ & 136.1085 \\
\hline $4-6$ & 95.1741 \\
\hline $5-6$ & \\
\hline
\end{tabular}

\subsubsection{Radio Propagation Energy Model}

To be able to simulate energy consumption in radio signal propagation, a model which enables simulation of parameters of interest was selected. The radio energy model used in this project was proposed by (Reusens E. et al, 2009). This model uses the distance (d) between the transmitter and the receiver and assumes the loss of energy due to transmission channel $\left(\mathrm{d}^{2}\right)$. The model equations are shown below:

$\mathrm{E}_{\mathrm{Tx}}(\mathrm{k}, \mathrm{d})=\mathrm{E}_{\mathrm{Tx}}-$ elec $(\mathrm{k})+\mathrm{E}_{\mathrm{Tx}}-{ }_{\text {amp }}(\mathrm{k}, \mathrm{d})$

$\mathrm{E}_{\mathrm{Tx}}(\mathrm{k}, \mathrm{d})=\mathrm{E}_{\mathrm{Tx}}-{ }_{\text {elec }} \mathrm{x} \mathrm{k}+\mathrm{E}_{\mathrm{amp}} \mathrm{x} \mathrm{kx} \mathrm{d} \mathrm{d}^{2}$ 
International Journal of Wireless \& Mobile Networks (IJWMN) Vol. 7, No. 5, October 2015

$\mathrm{E}_{\mathrm{Rx}}(\mathrm{k})=\mathrm{E}_{\mathrm{Rx}}{ }^{-{ }_{\text {elec }}}(\mathrm{k}) \mathrm{E}_{\mathrm{Rx}}(\mathrm{k})=\mathrm{E}_{\mathrm{Rx}{ }^{-} \text {elec }} \mathrm{x} \mathrm{k}$

$\mathrm{E}_{\mathrm{Rx}}(\mathrm{k})=\mathrm{E}_{\mathrm{Rx}}-{ }_{\text {elec }} \mathrm{x} \mathrm{k}$

Where;

$\mathrm{E}_{\mathrm{Tx}}$ is transmission energy in Joules

$\mathrm{E}_{\mathrm{Rx}}$ is energy consumed by the receiver in Joules

$\mathrm{E}_{\mathrm{Tx}}-$ elec are energies required by the circuitry of transmitter and receiver in $\mathrm{nJ} / \mathrm{bit}$.

$\mathrm{E}_{\mathrm{amp}}$ is the energy required by the amplifier circuit in $\mathrm{J} / \mathrm{bit}$.

$\mathrm{k}$ is the packet size in bits

Since in WBAN there is attenuation due to path loss, a path loss coefficient parameter $n$ is added to the equation above thus can be written as:

$\mathrm{E}_{\mathrm{Tx}}(\mathrm{k}, \mathrm{d})=\mathrm{E}_{\text {elec }} \times \mathrm{k}+\mathrm{E}_{\text {amp }} \times n \times \mathrm{k} \mathrm{x} \mathrm{d}^{n}$

The parameters in the above equations depend on the type of transceivers used. There are two transceivers commonly used in WBANs, Nordic nF 2401A and Chipcon CC2420. Nordic nF $2401 \mathrm{~A}$ is a single chip and consumes low power. The bandwidth of both transceivers is $2.4 \mathrm{Ghz}$. Nordic $\mathrm{nF} 2401 \mathrm{~A}$ transceiver is chosen for this project because its low power consumption. The parameters of the two transceivers are given in table 5 below

Table 2: Radio Parameters of Nordic nF 2401A Transceiver

\begin{tabular}{l|lll} 
Parameter & nF 2401A & CC2420 & Units \\
\hline DC Current(Tx) & 10.5 & 17.4 & $\mathrm{~mA}$ \\
DC Current(Rx) & 18 & 19.7 & $\mathrm{~mA}$ \\
Supply Voltage(min) & 1.9 & 2.1 & $\mathrm{~V}$ \\
$\mathbf{E}_{\mathrm{Tx} \text { - elec }}$ & 16.7 & 96.9 & $\mathrm{~nJ} / \mathrm{bit}$ \\
$\mathbf{E}_{\mathrm{Rx} \text { - elec }}$ & 36.1 & & $\mathrm{~nJ} / \mathrm{bit}$ \\
$\mathbf{E}_{\text {amp }}$ & $1.97 \mathrm{e}-9$ & 172.8 & $\mathrm{~J} / \mathrm{bit}$
\end{tabular}




\section{3 Simulation Model}

The simulation was done using Simulink. The simulation model is as shown below.

Figure 3: Simulation Model

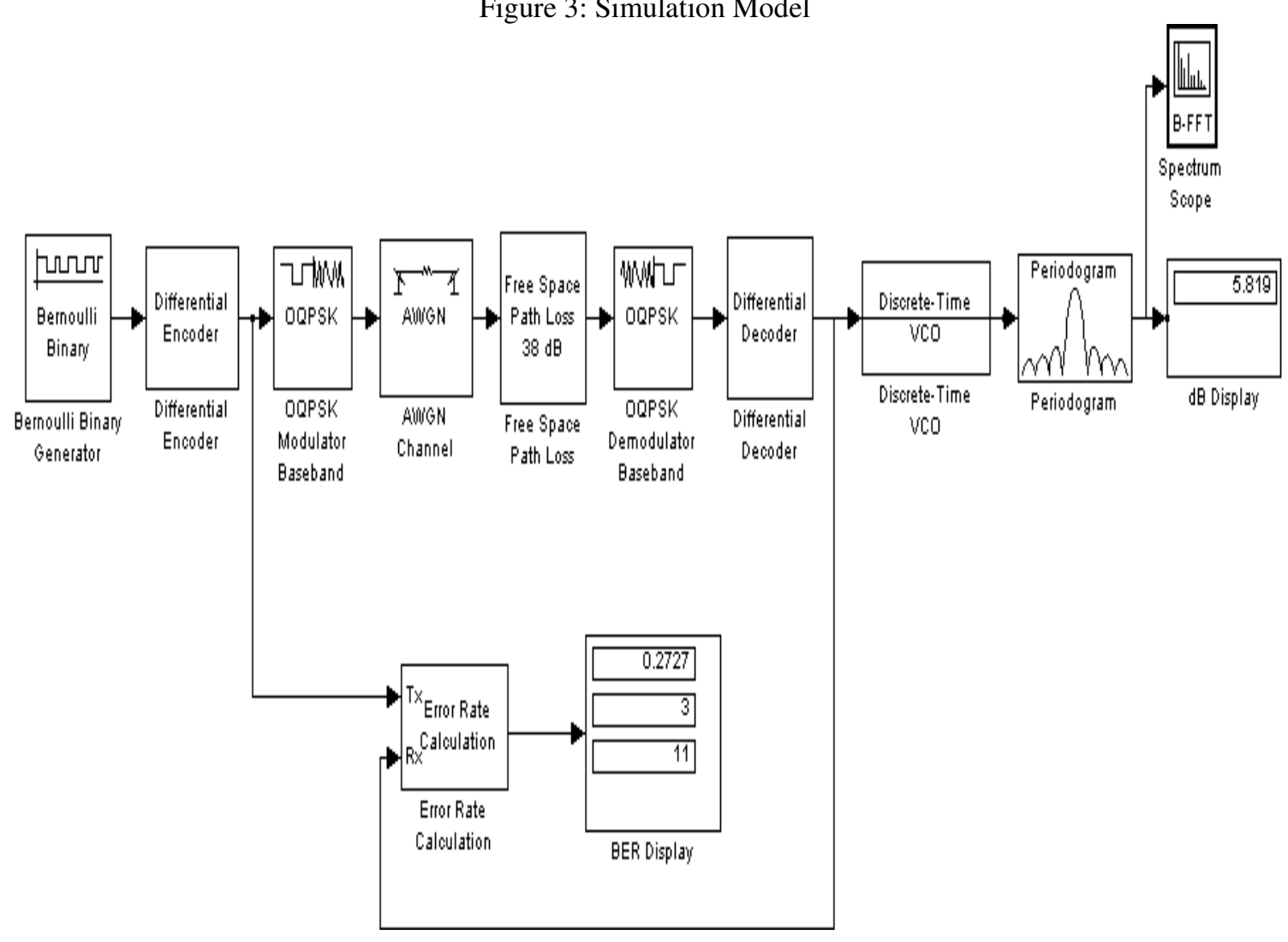

\subsubsection{Components of the Simulation Model}

\subsubsection{Bernoulli Binary Generator}

The Bernoulli Binary Generator block uses the Bernoulli distribution to randomly produce binary numbers. The Bernoulli distribution with parameter $\mathrm{p}$ produces zero with probability $\mathrm{p}$ and one with probability 1-p. The Bernoulli distribution has mean value 1-p and variance $p(1-p)$. The Probability of a zero parameter specifies $\mathrm{p}$, and can be any real number between zero and one.

\subsubsection{Differential Encoder}

The Differential Encoder block performs signal encoding on the binary input signal. The output is the logical difference between the present input and the previous output. The input can be either a scalar or a vector. The initial output value is formed by getting the logical exclusive-OR of this value with the initial input value. 
International Journal of Wireless \& Mobile Networks (IJWMN) Vol. 7, No. 5, October 2015

\subsubsection{OQPSK Modulator Baseband}

The OQPSK Modulator Baseband block is used to perform signal modulation. It uses offset quadrature phase shift keying method to execute this process. Baseband modulation and demodulation techniques are fundamental to communication systems. Baseband is actual frequency band of signal (e.g voice, video). Modulation enables the signal to be transmitted by a carrier frequency.

It receives the encoded signal from the binary encoder before the signal is released to the transmission channel.

\subsubsection{AWGN Channel}

This block represents a typical transmission channel by adding interference to the signal. The block adds white noise the signal so that effects of the interference can be captured.

The relative power of noise in an AWGN channel is typically described by quantities such as

- $\quad$ Signal-to-noise ratio (SNR) per sample. This is the ratio of the signal strength to that of noise.

- Ratio of bit energy to noise power spectral density (Eb/No). This quantity is used by BERTool and performance evaluation functions in this toolbox.

- $\quad$ Ratio of symbol energy to noise power spectral density (Es/No)

The relationship between Es/No and Eb/No, both expressed in $\mathrm{dB}$, is as follows:

$\mathrm{E}_{\mathrm{S}} / \mathrm{N}_{\mathrm{O}}(\mathrm{dB})=\mathrm{E}_{\mathrm{b}} / \mathrm{N}_{\mathrm{O}}(\mathrm{dB})+10 \log _{10}(\mathrm{k})$

Where $\mathrm{k}$ is the number of information bits per symbol.

The relationship between Es/No and SNR, both expressed in $\mathrm{dB}$, is as follows:

\subsubsection{Free Space Path Loss}

The Free Space Path Loss block represents channel parameters which affects signal transfer. The distance between the receiver and transmitter is the main variable affecting signal power loss. The block varies the amplitude of the received signal element by a value determined by carrier frequency and distance or loss parameter when decibels is used.

In this simulation, distance and carrier frequency parameters are used since distance between the nodes was calculated and the frequency for ISM band is $2.4 \mathrm{GHz}$.

This block accepts a column vector input signal. The input signal to this block must be a complex signal. The reciprocal of the loss is applied as a gain, e.g., a loss of $+20 \mathrm{~dB}$, which reduces the signal by a factor of 10 corresponds to a gain value of 0.1 .

\subsubsection{OQPSK Demodulator Baseband}

The OQPSK Demodulator Baseband block is used in signal demodulation. The block takes in the baseband representation of the input signal and demodulates it. 
International Journal of Wireless \& Mobile Networks (IJWMN) Vol. 7, No. 5, October 2015

The block outputs integer symbol values from 0 to 3 or 2-bit binary representation of the integers depending on the set type of output parameter.

\subsubsection{Differential Decoder}

This block plays a very important role in converting binary input bit to equivalent signal element which can be transmitted through the channel.

\subsubsection{Bit Error Rate Calculation}

This block calculates the rate of error occurrence in the received signal. It does this by comparing transmitter output data and receiver data output.

The output of this block is normally connected to the bit error display block.

\subsubsection{Bit Error Display}

The Display block is used to show the bit errors in the signal received calculated in the bit error calculation block.

The output is displayed in three rows. The upper row shows the error rate, the middle row shows the error bits and the lower row displays the number of comparisons.

\subsubsection{Display}

It shows the data of its input on its display icon.

\subsubsection{Spectrum Scope}

This block is used to calculate and display outputs at various times of the input. The input can be sampled or frame-based matrix or vector.

This block is used to produce a spectrum of the signal. It can produce time domain spectrum or frequency spectrum.

\subsubsection{Voltage Controlled Oscillator}

This block produces a signal which shifts from the optimum frequency with a value proportional to the input signal.

This block produces an output signal which can be used to represent the magnitude of a signal.

\subsubsection{Periodogram Block}

This block calculates an approximation components of the spectrum which not parameters. It is used to show the graphical variation of an input over time at different times. 
International Journal of Wireless \& Mobile Networks (IJWMN) Vol. 7, No. 5, October 2015

\section{DISCUSSION OF FINDINGS}

\subsection{Discussion of Findings}

From the results of the simulation it is evident that path loss varies upwards with increase in distance. Figure 7 in the previous section shows a steady rise in path loss with increase in distance between the transmitter and the receiver. This path loss in effect leads to data loss which is not desirable in WBANs. When a relay is used, the interference on the signal due to free space path loss is reduced thus enhancing energy reduction during transmission as well as ensuring data signals are not lost.

Node placement in relation to coordinator and relay can be varied to achieve optimal distance separating the transmitter and receiver.

The RSSI parameter can be used to check the signal strength. From the simulation model, a peridogram is used to capture this on magnitude verses frequency plot as shown below.

\subsection{Performance Metrics}

\subsubsection{Path Loss Calculation}

Path loss shows the relative strength between the transmitted power at the sending node and received power at the receiver.

The path loss between the sending and the receiving antenna was calculated as a function of distance parameter using the formula described earlier in equation (1):

$\mathrm{PL}(\mathrm{d})=\mathrm{PL}_{0}+10 \mathrm{n} \log _{10}\left(\mathrm{~d} / \mathrm{d}_{0}\right)$

The path loss can also be calculated as function of both distance and frequency using the formula

$\mathrm{PL}=20 \log _{10}(4 \pi \mathrm{df} / \mathrm{c})$

Where $\mathrm{d}$ is the distance between the transmitter and receiver, $\mathrm{f}$ is the frequency and $\mathrm{c}$ is the speed of light. In free space, path loss coefficient $n$ used is 3.38 since LOS is assumed in this work (Elias J and Mahaoua A, 2012)

In WBAN $n$ varies from 3 to 4 for line of sight (LOS) transmission and 5 to 7.4 for non line of sight (NLOS) transmission.

The distance was measured between the node and the relay as well as the coordinator. In this investigation the $2.4 \mathrm{GHz}$ frequency is used as it is freely available and it has been a choice of many researchers in WBAN designs in this band. The path loss exponent in free space is two (2) as highlighted by (Reusens E. et al, 2009). 
International Journal of Wireless \& Mobile Networks (IJWMN) Vol. 7, No. 5, October 2015

MATLAB was used to randomly generate sensor nodes within a range of $150 \mathrm{~cm}$ and algorithm to allow connection between the sensor and the coordinator if the distance is less than or equal to the predetermine distance $(\mathrm{R})$ otherwise the relay is used to send sensor data to the coordinator. In this case the optimum distance chosen was $75 \mathrm{~cm}$, though this can be varied.

Table 3: Path Loss and Distance Data from MATLAB

\begin{tabular}{|c|c|}
\hline 0.10 & -45.9559 \\
\hline 10.10 & 46.3465 \\
\hline 20.10 & 60.1102 \\
\hline 30.10 & 68.1863 \\
\hline 40.10 & 73.9233 \\
\hline 50.10 & 78.3762 \\
\hline 60.10 & 82.0160 \\
\hline 70.10 & 85.0942 \\
\hline 80.10 & 87.7613 \\
\hline 90.10 & 90.1142 \\
\hline 100.10 & 92.2192 \\
\hline 110.10 & 94.1236 \\
\hline 120.10 & 9515623 \\
\hline 130.10 & 97.4618 \\
\hline 140.10 & 98.9429 \\
\hline
\end{tabular}

A plot of path loss against distance depicted an increase in path loss with distance as shown in the figure below: 
International Journal of Wireless \& Mobile Networks (IJWMN) Vol. 7, No. 5, October 2015

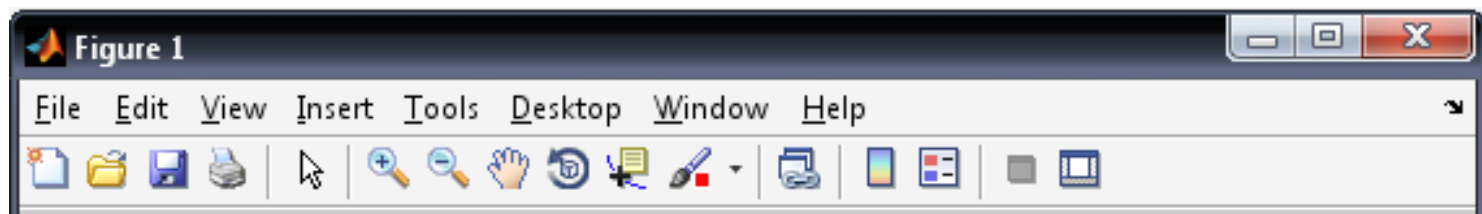

Path loss verses Distance between $\mathrm{TX}$ and $\mathrm{RX}$

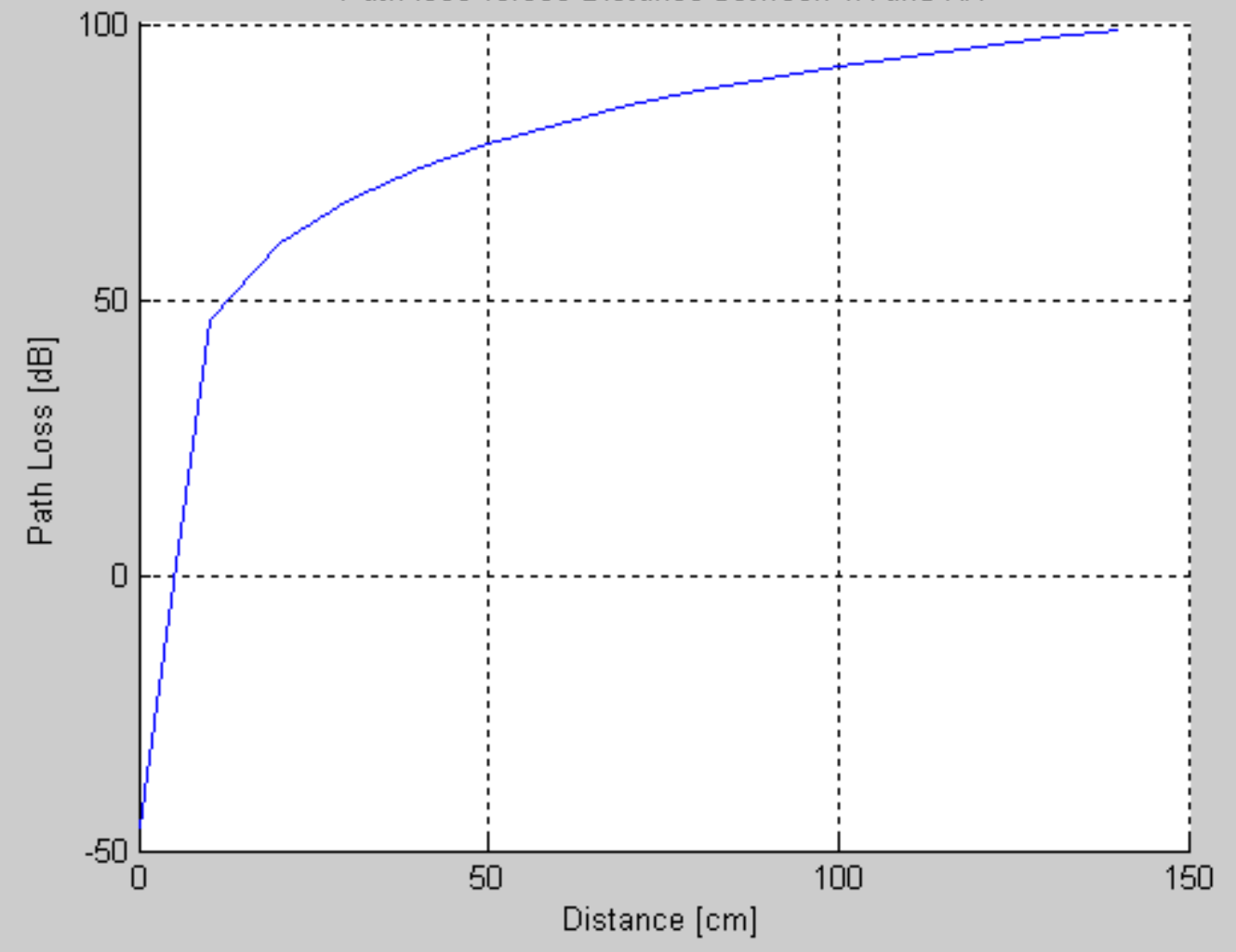

Figure 4: Plot of Path Loss versus Distance Chart

\subsubsection{Energy Dissipation of the radio model}

The energy required to transmit data at a rate of $250 \mathrm{~Kb} / \mathrm{s}$ for a period of 0.01 seconds was investigated. 
International Journal of Wireless \& Mobile Networks (IJWMN) Vol. 7, No. 5, October 2015

\subsubsection{Transmitter Energy}

Table 4: Transmitter Energy and Distance Data from MATLAB

\begin{tabular}{|ll|}
\hline Distance (cm) & Transmitter Energy (nJ) \\
\hline 10 & 0.0061 \\
\hline 20 & 20.9859 \\
\hline Bo & 257.9027 \\
\hline 40 & $1.1688 \mathrm{e}+003$ \\
\hline 50 & $3.4581 \mathrm{e}+003$ \\
\hline 60 & $8.0651 \mathrm{e}+003$ \\
\hline 70 & $1.6155 \mathrm{e}+004$ \\
\hline $\mathbf{8 0}$ & $2.9115 \mathrm{e}+004$ \\
\hline 90 & $4.8547 \mathrm{e}+004$ \\
\hline 100 & $7.6264 \mathrm{e}+004$ \\
\hline 110 & $1.1429 \mathrm{e}+005$ \\
\hline 120 & $1.6485 \mathrm{e}+005$ \\
\hline 130 & $2.3038 \mathrm{e}+005$ \\
\hline 140 & $3.1351 \mathrm{e}+005$ \\
\hline & $4.1707 \mathrm{e}+005$ \\
\hline
\end{tabular}


International Journal of Wireless \& Mobile Networks (IJWMN) Vol. 7, No. 5, October 2015

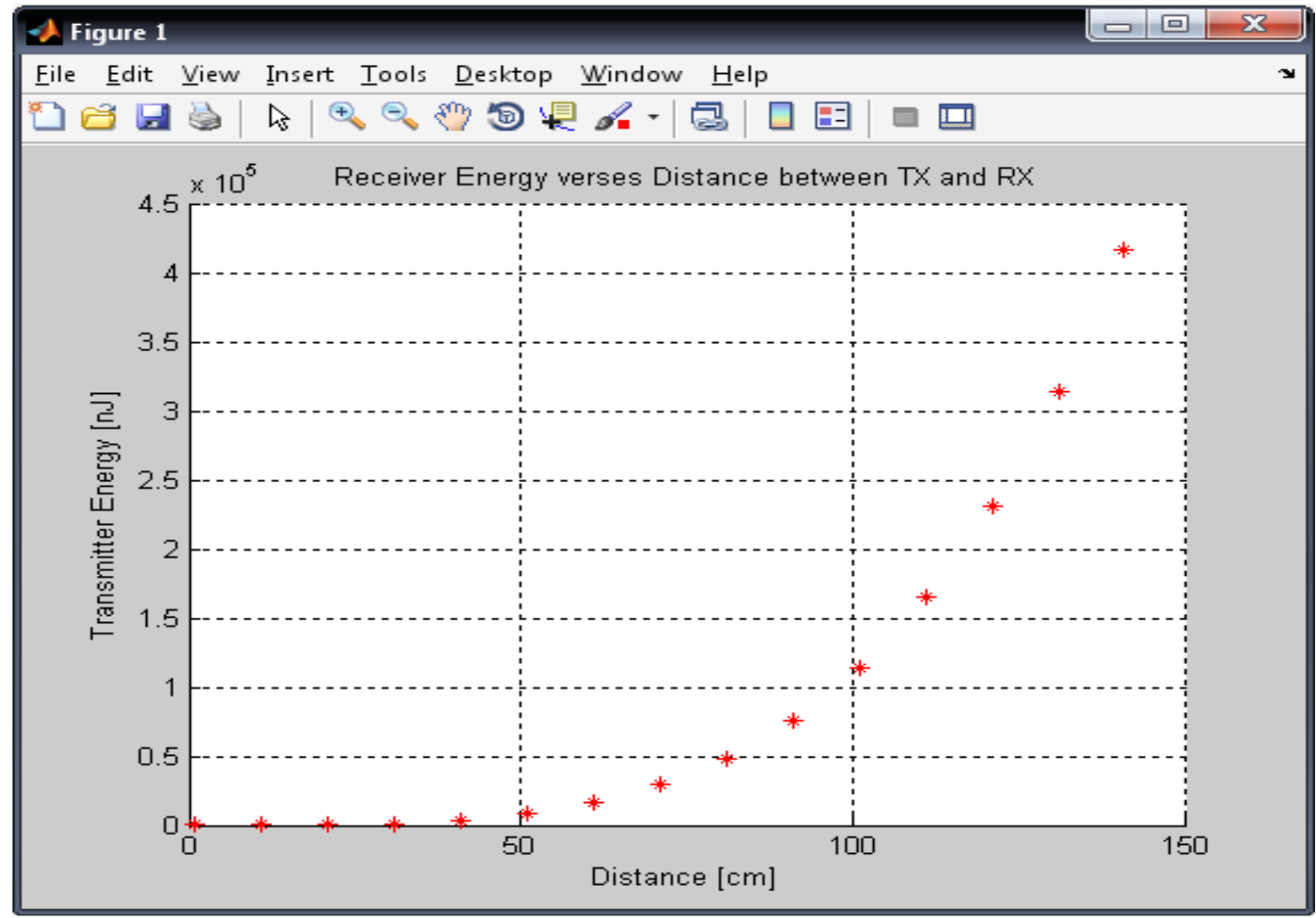

Figure 5: Plot of Transmitter Energy verses Distance

\section{CONCLUSION AND FURTHER WORK}

\subsection{Conclusion}

In this research project, we propose an energy efficient model for deploying WBAN using multi hop topology. Use of a relay between the sensor nodes and the coordinator optimally placed is suggested. The model uses an optimum distance to place a relay for retransmitting signals from nodes beyond the specified distance. The simulation showed that the nodes uses lower energy to send signals through a relay.

The energy consumption graph showed that the relay can be placed at a distance of between $50 \mathrm{~cm}$ and $75 \mathrm{~cm}$ to achieve energy efficiency.

Path loss was used as a performance metric and it showed that the further the nodes from the coordinator, the higher the path loss. Use of the relay reduces path loss. However, the relay also adds to the cost of system design since it will require higher hardware specifications to be able to transmit signal from the nodes. This challenge can be solved by replacing relay batteries periodically when they get depleted. 
International Journal of Wireless \& Mobile Networks (IJWMN) Vol. 7, No. 5, October 2015

The transmitter energy required also was shown to increase with distance. This implies that when a relay is used, lower energy will be dissipated during signal transmission.

\subsection{Further Work}

This area of energy efficiency in WBAN is of utmost importance in deploying WBAN for medical and health monitoring. In this research, the effect of distance between the receiver and transmitter on power consumption was the main variable investigated. The receiver signal strength indicator can be used to check the power of the signals at the receiver.

\section{REFERENCES}

[1] Min Chen et. al, "Body Area Networks: a Survey", Mobile Networks and Applications The Journal of SPECIAL ISSUES on Mobility of Systems, Users, Data and Computing, Mobile Netw Appl (2010) 16:171-193

[2] Wout J. etal "Design of Energy Efficient Topologies for Wireless On-Body Channel" European Wireless 2011, April 27-29, 2011, Vienna, Austria

[3] Majid N et al, "A Robust Protocol Stack for Multi-hop Wireless Body Area

Networks with Transmit Power Adaptation," BodyNets'10 September 10-12, 2010, Corfu Island, Greece.

[4] Al Ameen et al, "A power efficient MAC protocol for wireless body area networks," EURASIP Journal on Wireless Communications and Networking 2012, 2012:33, http://jwen.eurasipjournals.com/content/2012/1/33

[5] Reusens E. et al, (2009). "Characterization of On-Body Communication Channel and Energy Efficient Topology Design for Wireless Body Area Networks", IEEE Transactions on Information Technology in Biomedicine, Vol. 13, No. 6, November 2009

[6] Mehmet R. Yuce and Jamil Khan., Wireless Body Area Networks: Technology, Implementation, and Applications, Taylor \& Francis, 2011.

[7] Meenakshi Bansal and Navroop Kaur, "Use of Relay Nodes in Body Area Sensor Network for Reducing Power Consumption" IJCST Volume 2, Issue 1, March 2011

[8] Navid A. et al, "Joint consideration of energy-efficiency and coverage-preservation in microsensor networks", Journal of Wireless Communications and Mobile Computing 2011; 11:707-722

[9] Qutaibai et al, (2012) "Simulation Framework of Wireless Sensor Network (WSN) Using MATLAB/SIMULINK Software", Iraq Journal of Electrical and Electronic Engineering Vol.7, No. 2, 2011

[10] Mahlknecht S. Madani S. and Kazmi J. (2010). "Wireless Sensor Networks: Modeling and Simulation, Discrete Event Simulations", Aitor Goti (Ed.), ISBN: 978-953-307-115-2, InTech

[11] Nurul I. and Syafnidar A. (2011). "A Review of Simulation of Telecommunication Networks: Simulators, Classification, Comparison, Methodologies and Recommendations", Journal of Selected Areas in Telecommunications (JSAT), March Edition, 2011

[12] Rune J. et al, (2011). "A Modular Platform for Wireless Body Area Network Research and Real-life Experiments", International Journal on Advances in Networks and Services, vol. 4 no 3 \& 42011.

[13] Garth V. et al, (2012). "Wireless Body Area Networks for Healthcare: A Survey", International Journal of Ad hoc, Sensor \& Ubiquitous Computing (IJASUC) Vol.3, No.3, June 2012

[13] Shahnaz Saleem, Sana Ullah and Kyung Sup Kwak, "A Study of IEEE 802.15.4 Security Framework for Wireless Body Area Networks" Article, Sensors 2011

[14] Laurie Hughes. Et al, (2012). "A Review of Protocol Implementations and Energy Efficient CrossLayer Design for Wireless Body Area Networks" Sensors 2012, 12, 14730-14773; doi:10.3390/s121114730 
International Journal of Wireless \& Mobile Networks (IJWMN) Vol. 7, No. 5, October 2015

[15] Al Ameen et al, (2012). "A power efficient MAC protocol for wireless body area networks", EURASIP Journal on Wireless Communications and Networking 2012

[16] Javaid N. et al, (2013). "Ubiquitous HealthCare in Wireless Body Area Networks-A Survey", Journal of Basic Applied Scie.

[17] Elias J and Mahaoua A, (2012) "Energy-aware Topology Design for Wireless Body Area Networks" Selected Areas in Communications Symposium, IEEE ICC 2012.

[18] Md.Asdaque Hussain and Kyung Sup Kwak, "Positioning in Wireless Body Area Network using GSM," International Journal of Digital Content Technology and its Applications Volume 3, Number 3, September 2009.

[19] Mark Andrew Hanson, Wireless Body Area Sensor Network Technology for Motion-Based Health Assessment, 2nd ed., BiblioBazaar, 2010.

[20] Khalid Abu Al-Saud et al, "Wireless Body Area Sensor Networks Signal Processing and Communication Framework: Survey on Sensing, Communication Technologies, Delivery and Feedback," Journal of Computer Science 8 (1): 121-132, 2012.

\section{Authors}

\section{Robert C. Chepkwony}

Msc in Data Communication from KCA University in 2013. Graduated with Bachelor of Technology Education (Electrical and Electronics) from Moi University in 2005. Have taught in various Universities and currently working as a Regional Centre Coordinator in The Digital School at Kenyatta University - Nairobi Kenya.

\section{John O. Gwendo}

Master of Science in Information Systems (University of Nairobi) -2011, Bachelor of Technology Education- Electrical \& Electronics (Moi University)-2002, Certified Information Communication Technologist - KASNEB-Kenya-2010, Diploma - Institute for the Management of Information Systems- Strathmore College-1998. Experience: I have taught at an Institute of Science and Technology and a number of Universities in Kenya. Currently Lecturing at Moi University- Eldoret, Kenya.

\section{Peter K. Kemei}

Mr. Peter K. Kemei Msc, Bsc, Dip Electrical Eng, CCNA and A+ Certified. Teaching at Egerton University, Kenya for more than 12 years and offering consultancy services in Computer Networking \& Data Communications, Hardware and Software Maintenance, Network Security, Mobile Computing and Wireless Currently my interested revolves around theoretical, practical and research work centered on network security and Data Communication related areas.

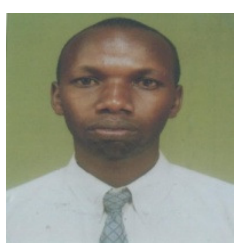

